\title{
ACADÉMIE INTERNATIONALE DE CYBERNÉTIQUE ET SCIENCE DES SYSTÈMES : PREMIÉRE ATTRIBUTION DU PRIX INTERNATIONAL CHARLES FRANÇOIS
}

\author{
Pierre BRICAGE \\ Secrétaire général Académie Internationale de Cybernétique et Science des Systèmes IASCYS \\ http://iascys.org \\ Long Term Guest Professor University of Sichuan, Chengdu, P.R. China \\ http://web.univ-pau.fr/ bricage \\ Vice-Président Association Française de Science des Systèmes Cybernétiques Cognitifs et Techniques AFSCET \\ http://www.afscet.asso.fr/pagesperso/Bricage.html \\ pierre.bricage@univ-pau.fr
}

\begin{abstract}
Résumé :
L'Académie Internationale de Cybernétique et Sciences des Systèmes, IASCYS, association sans but lucratif et ne disposant d'aucun financement, décerne, sans périodicité, le Prix International Charles François dont l'un des objectifs est de relier les structures et les compétences en cybernétique et systémique, quels que soient la langue et le domaine d'expertise des acteurs. Décerné pour la première fois, après un processus de pré-sélection anonyme et d'évaluation consensuelle, le prix a récompensé, dans des domaines variés (Cybersystemic Management of Changes, Professional Methods and Tools for Cybersystemics), à la fois des chercheurs confirmés mais 'oubliés 'et de jeunes chercheurs 'prometteurs'.
\end{abstract}

Mots-clés :

ateliers, congrès, IASCYS, IFSR, Prix International, relier, science des systèmes, talents

\section{ABOUT THE FIRST AWARDS OF THE INTERNATIONAL PRIZE CHARLES FRANÇOIS OF THE INTERNATIONAL ACADEMY FOR SYSTEMS AND CYBERNETIC SCIENCES}

\section{Extended Abstract:}

For the first time the International Academy for Systems and Cybernetic Sciences, IASCYS, (http://iascys.org), has awarded the Charles Francois International Prize, during the $10^{\text {th }}$ UES-EUS Congress (http://ues-eus.eu), in Brussels, Belgium, Europe.

The first step of the procedure was the nomination of interesting papers through asking for the reviewing process by all IASCYS Academicians. So, 6 weeks before the start of the meeting, a booklet of 40 abstracts, all previously anonymously peer-reviewed by the scientific committee of the congress, and each as a 1 page of text, with neither author(s) name(s), nor affiliation(s) or references, was sent to all Academicians. After a 1 month delay, 10 papers of people from 9 Countries (Algeria, Belgium, Denmark, France, Germany, Greece, Italy, Portugal, Russia), have been nominated by Academicians, by e-mails replies. After the congress organizers have proposed as jurors a team of 3 systems scientists who all are speaking both French and English (the official formal languages of the UES-EUS congress), an equal number of 3 Academicians, who are as well fluent in English, French and other languages, attended as the IASCYS part of the jury. After this key step of peers preselection, the second step, during the meeting in Brussels, was for all 6 jurors to listen and participate to the corresponding talks-debate for each of the selected papers, in order to rank the top 3 of the most promising works, and then to reflect on the final ranking for the award. The jurors were anonymous. The listening process was the usual process of talk (20 min) and questions (10 min) with the public as in every congress, but also with personal no-formal discussion of jurors with the nominated persons.

The first Charles François International Prize of the Academy (gold medal) was awarded to Julio LABORDE, a young Chilean research engineer who is working in the International industrial firm 'Insight Signals'. He is also a student in the prestigious École Pratique des Hautes Études, in Paris, France. His talk was about "Extraction of Information from Agent Base Models. A new pre-topological metric for controlling the propagation of crises." It took place during the 'Methods and tools for risk management of complex socio-technical systems' session. No discussion, his work was the most promising work of the congress. His work was the most promising work of this congress of the European Union for Systemics. 
This second step allows also, after a debate, to award 3 second places ( 3 silver medals). They all got the Charles François tutorial in Systems Science on a USB stick and they all, gold and silver medals (Figure 1), will have a certificate of ranking.

But, all of them, will get their certificates of award/ranking only after their paper proof will be corrected and accepted. The other 3 certificated persons, silver medals, are (by alphabetic order): -Mick ASHBY, a research engineer in computing sciences who is working for IBM in Germany. His work was about the application of a new paradigm 'The Ethical Regulator Theorem'; -Tjorven HARMSEN, a very young Swedish women who is in Ph. D. in the Leibniz Institute for Research on Society and Space, in Berlin (Germany). Her work was about 'Crisis as Social Autocatalysis. On the emergence and Utilization of Opportunities' (a very promising talk, a young researcher to follow; and-Daniela TERRILE, a women who is Professor in the Department of Design at the Polytechnic Institute of Milano, Italy. Her work was about 'Applications of the Target Constellation Model'. 2 other works were also very interesting but one was not into the book of abstracts (it was not peer-reviewed), the other one was into it but it was not pre-selected. No process is perfect. But the rule is the rule: no peerreviewing, no preselection, means no competition. The important point is that few of the 'preselected and nominated, but not ranked in the top 3' participants said they will attend the next one edition of the Prize in Morocco. And other young researchers said they will do their best to attend another occurrence of the Prize.

The Prize was opened to strengthen multi-disciplinary research and the multi-language communication of recent results, towards a worldwide education in Cybernetics and Systems Thinking (Bricage, 2017), which are aims of the Academy (Bricage, 2014). "Want to influence the world? Map reveals the best languages to speak." (Ronen et al., 2014). On the IASCYS website you will find the rules of the Prize, in English, Spanish, French and Russian.

Key words:

congress, giftedness, IASCYS, IFSR, International Prize, reliability, reliance, Systems Science, workshops

L'Académie Internationale de Cybernétique et Sciences des Systèmes, IASCYS, a été fondée en 2010 par l'International Federation for Systems Research (IFSR, Vienne, Autriche, http://ifsr.org). Elle est enregistrée en France, comme association loi 1901, depuis le 14 juillet $2016^{1}$. Elle a pour objet non seulement d'honorer d'éminents chercheurs mais surtout de tenter de resserrer les liens, de faciliter les interactions, entre les organisations oeuvrant de façon trans-disciplinaire dans les champs de la cybernétique et des sciences des systèmes ${ }^{2}$ (Bricage, 2014). C'est le but de ce prix Charles François. L'académie a aussi pour objet d'encourager et d'organiser des actions d'éducation (Bricage, 2017), quel que soit le niveau des participants, et d'organiser des ateliers de travail lors de congrès nationaux ou internationaux. Elle ne dispose d'aucun financement. Les Académiciens sont recrutés sur concours. L'Académie fonctionne sur le bénévolat, en respectant l'éthique de responsabilité sociétale $e^{3}$ et aucune obligation n'est requise de la part de ses membres, sinon de respecter cette "cyber-règle": 'Academicians may not injure the Academy and its Partners or, through inaction, allow the Academy being to come to harm.'.

\section{UN PRIX INTERNATIONAL POUR QUOI ?}

Charles FRANÇOIS, Académicien fondateur de l'IASCYS et éditeur de l'Encyclopédie Internationale de Science des Systèmes, ouvrage en anglais (François, 2004), est d'origine belge, donc francophone, mais il a vécu dans de nombreux pays et s'est retiré en Argentine, pays hispanophone. Si on observe la distribution mondiale des travaux de recherche publiés ces dix dernières années (Ronen et al., 2014),

\footnotetext{
${ }^{1}$ République Française : RNA n ${ }^{\circ} \mathbf{W 7 6 3 0 1 3 1 1 4}$, Yearbook of International Organizations: uia.org J5273

2 What about systems science and cybernetics? (English .pdf file)

Sistemas ciencia. Aprehender un mundo globalizado. (texto .pdf en español)

L'approche systémique. Appréhender la globalité. (texte .pdf en français)

${ }^{3}$ Systemic behaviour via the principles of Social Responsibility (alphabetic order): accountability, ethical behaviour, fair governance, fair operating practices, interdependence and holism, respect for the rules, transparency (norme ISO 26000).
} 
on constate que les 3 langues, anglais, espagnol et français, permettent d'atteindre plus des $2 / 3$ des locuteurs.

La journée mondiale de la traduction ${ }^{4}$, en 2018 à Pau, France, l'a encore rappelé, la diversité culturelle européenne est d'abord une diversité linguistique.

\section{La diversité culturelle et linguistique est la variété pré-requise de la survie de l'Union Européenne.}

Ce prix est décerné uniquement à l'occasion de congrès conjoints, trans-disciplinaires, organisés par des associations membres des réseaux de l'UES-EUS (Bruxelles, Belgique, http://ues-eus.eu), du WOSC (Lincoln, UK, http://wosc.co), de l'IFSR, ou d'autres structures nationales ou internationales, comme l'IEEE (https://www.ieee.org), comme lors de la prochaine Word Conference on Complex Systems (WCCS, Maroc, http://mscomplexsystems.org/wccs19), ce sans limite et sans exclusion. L'objectif est relier les structures, de faire coopérer les Comités Exécutifs et de faire émerger des jeunes talents tout en confirmant les talents anciens parfois non reconnus.

L'Académie a déjà participé, en 2012 et 2014, à la remise conjointe du Bertalanffy Award for Young Scientists, organisé par le Bertalanffy Center for the Study of Systems Science (BCSSS, Vienna, Austria, http://www.bcsss.org) lors des Congrès de l'EMCSR (European Meetings on Cybernetics and Systems Research, http://emcsr.net), congrès organisés en coopération avec diverses organisations internationales. par l'Austrian Society for Cybernetic Studies (OSGK, http://www.osgk.ac.at/emcsr). A chaque occasion où elle intervient l'Académie délivre un certificat d'excellence aux lauréats, avec l'indication des Académiciens et des participants extérieurs à l'Académie.

\section{UN PRIX INTERNATIONAL COMMENT?}

\section{Comment fonctionne le jury ? Quelles sont les étapes de sélection ?}

La première étape est celle de la sélection par les Académiciens, à partir du livret des résumés des communications acceptées (qui ont donc déjà été revues et corrigées une première fois par le comité scientifique du congrès), de celles qui semblent les plus prometteuses pour certains Académiciens. Plus d'un mois avant le début du congrès les Académiciens reçoivent ce livret, actualisé, et clos, avec des résumés anonymes : seuls les titres accompagnent le texte du résumé, en anglais, en français ou/et en espagnol, ce sans aucune indication ni de noms d'auteurs, ni des affiliations des auteurs, et sans bibliographie. Cette étape peut aboutir, ou non, à une large sélection de travaux. Pour cette première édition, à Bruxelles, 10 communications avaient été retenues, d'auteurs provenant de 9 pays (Algérie, Allemagne, Belgique, Danemark, France, Grèce, Italie, Portugal, Russie), intervenant dans diverses sessions du congrès (Blockchain et Intelligence Artificielle : des Technologies de Rupture; Crises et Accompagnement du Changement; Methods and Tools for Risk Management of Complex SocioTechnical Systems; Professional Systemics).

La deuxième étape est l'étape traditionnelle de tout congrès. Chaque auteur pré-sélectionné, sans qu'il le sache, est évalué par les membres du co-jury IASCYS-organisateurs. Les jurés participent anonymement aux exposés et débats, qui peuvent se dérouler en anglais, français ou/et espagnol. Lorsque les documents projetés sont en français (langue du pays organisateur), ou en espagnol, l'exposé oral est en anglais et lorsque les documents projetés sont en anglais ou en espagnol, l'exposé oral est en français ou en anglais. D'autres langues peuvent être utilisées pour les documents projetés. Seuls les travaux dont les résumés ont été pré-sélectionnés sont évalués. Le meilleur exposé du travail le plus prometteur reçoit, à la fin du congrès, le prix Charles François sous forme d'un certificat d'excellence et les 2 volumes de l'Encyclopédie de Cybernétique et Science des Systèmes. ${ }^{5}$ Tous les travaux du top 3 (médailles de bronze, d'argent et d'or) reçoivent en outre le CD du tutoriel de Science des Systèmes édité par Charles François (François, 2007) sous forme d'une clé USB (Figure 1). Le

\footnotetext{
${ }^{4}$ Collectif JMT (28 septembre 2018). Journée Mondiale de la Traduction, Pau, France.

5 La deuxième édition, en anglais, en 2 volumes, est épuisée. L'Académie en possède encore quelques exemplaires, neufs, dédicacés par Charles François. La valeur de l'Encyclopédie à l'état neuf est d'environ 500 euros.
} 
prix et les accessits ne sont pas nécessairement délivrés. Cela dépend d'abord de la qualité des présentations orales et de l'intérêt potentiel des travaux présentés. Les lauréats doivent être présents pour recevoir leurs prix.

L'objectif est d'inciter les participants à participer à toutes les sessions et d'éviter le comportement du type « Je viens juste pour mon exposé, même si le congrès est déjà commencé depuis 1 ou 2 jours, et je pars aussitôt après, sans assister à d'autres sessions. Rien d'autre ne semble m'intéresser et je ne cherche pas à m'ouvrir à d'autres horizons. Je n'apprend rien... Je ne noue pas de liens. »

\section{La remise du Prix et les accessits}

Le prix final avec les certificats (Figure 1) attestant de l'excellence des travaux présentés n'est distribué (troisième étape) qu'après publication des travaux primés dans la revue du choix des organisateurs. Mais, à la condition suivante... Compte tenu de la diversité et de la spécificité professionnelle du parcours international de Charles François, compte tenu de la diversité linguistique des Académiciens, le texte écrit final doit être au moins bi-lingue, voire tri-lingue. Par exemple le texte complet peut être en anglais avec un résumé très étendu en français, ou l'inverse. Le texte en français, ou en anglais, peut être accompagné par un résumé étendu en anglais, ou en français, voire en espagnol, ou dans une autre langue (allemand, arabe, chinois, portugais, russe,...).

Toutes les instructions, actuellement en anglais, français, espagnol et russe, sont disponibles en ligne sur la page d'accueil du site de l'Académie. ${ }^{6}$

\section{UN PRIX INTERNATIONAL POUR QUI ?}

Deux chercheurs confirmés ont obtenu un accessit (médaille d'argent) :

- Daniela TERRILE ${ }^{7}$, qui est professeur de design à l'institut polytechnique de Milan, (Italie), et coach professionnelle (business practice coaching) à Bruxelles (Belgique), pour son travail 'Applications of the Target Constellation Model', dans la session 'Professional Systemics', et

- Mick ASHBY ${ }^{8}$ qui est ingénieur de recherche chez IBM en Allemagne. Mick a étudié les systèmes informatiques et les microprocesseurs à l'Université d'Essex, à Colchester, en Angleterre, UK, où il a étudié la logique pour gérer l'incertitude. Il a commencé à travailler pour ITT, au centre d'assistance technique du groupe de systèmes basés sur la connaissance, à Harlow, en Angleterre, où il a développé un langage d'intelligence artificielle permettant d'écrire des systèmes experts pour automatiser

\footnotetext{
${ }^{6}$ Charles Francois International Prize (English -.pdf file-), Premio Internacional Charles Francois (texto en español), Prix international Charles Francois (texte en français), Международный приз Шарля Франсуа (tексt по-русски)

${ }^{7}$ Daniela TERRILE daniela.terrile@gmail.com

Daniela TERRILE is professor at PoliDesign (Politecnico di Milano) and professional coach. HR accredited internal coach, ACC ICF coach, she is working as executive, life and personal development coach. Her interests are in business practice, family and organizational constellation systemic coaching. She keeps systemic4all ${ }^{\circledR}$ methodology on practising on her client's strengths and weaknesses, empowering participants, students, managers to face fears and improve their own personal style. Daniela is thriving when coaching, respecting and adapting to her client's preferences; she keeps an eye on research to improve her understanding of people's needs, reveal insights, and inspire innovative solutions in future management and leadership. She will start a PhD at Politecnico on Management. People inspire Daniela; she strongly believes that we have all good vibes, insights and internal resources to be effective and resilient. Her motto is: coaching is not about fixing people; coaching is enhancing their potential, to gain their space in society and become what they dream to be.

${ }^{8}$ Mick ASHBY ethics@ ashby.de

Mick Ashby is a Trustee of the American Society for Cybernetics. He studied Computer and Microprocessor Systems at the University of Essex, Colchester, England, where he researched into logics for handling uncertainty. He started working for ITT, Engineering Support Center, Knowledge-Based Systems Group, in Harlow, England, developing an AI language for writing expert systems for automating highly-skilled tasks in the telecommunications domain, such as configuring large switching systems and planning the physical layout of telephone exchanges. In 1988, he transferred to Alcatel Software Research Center, in Stuttgart, Germany, which was the prime-contractor for the EU RACE IOLE research project consortium. As project manager, he coordinated the consortium's research into requirements and solutions for performing online functional extension of broadband telecom software systems. For the last 20 years he has worked in the IBM Germany Research and Development Lab in Böblingen. He designed the W. Ross Ashby Digital Archive website. In his spare-time he has been researching into the cybernetics of ethics and publishes the results on his personal web site www.ashby.de.
} 
des tâches hautement spécialisées dans le domaine des télécommunications, avec la configuration de grands agencements des centraux téléphoniques. En 1988, il a été transféré au Alcatel Software Research Center de Stuttgart, en Allemagne, qui était le principal contractant du consortium de projets de recherche EU RACE IOLE. En tant que chef de projet, il a coordonné les recherches du consortium sur les exigences et les solutions pour le fonctionnement des systèmes logiciels de télécommunication. Au cours des 20 dernières années, il a travaillé au laboratoire de recherche et développement d'IBM Allemagne, à Böblingen. Il a conçu le site Web des archives numériques 'W. Ross Ashby'. Pendant son temps libre, il a effectué des recherches sur la cybernétique de l'éthique et en a publié les résultats sur son site personnel www.ashby.de. Chercheur confirmé, Mick est aussi administrateur de l'American Society for Cybernetics (ASC). Son travail récompensé portait sur la mise en oeuvre d'un nouveau paradigme: 'The Ethical Regulator Theorem', dans la session 'Crises, Cybersystemic Management of Changes'.

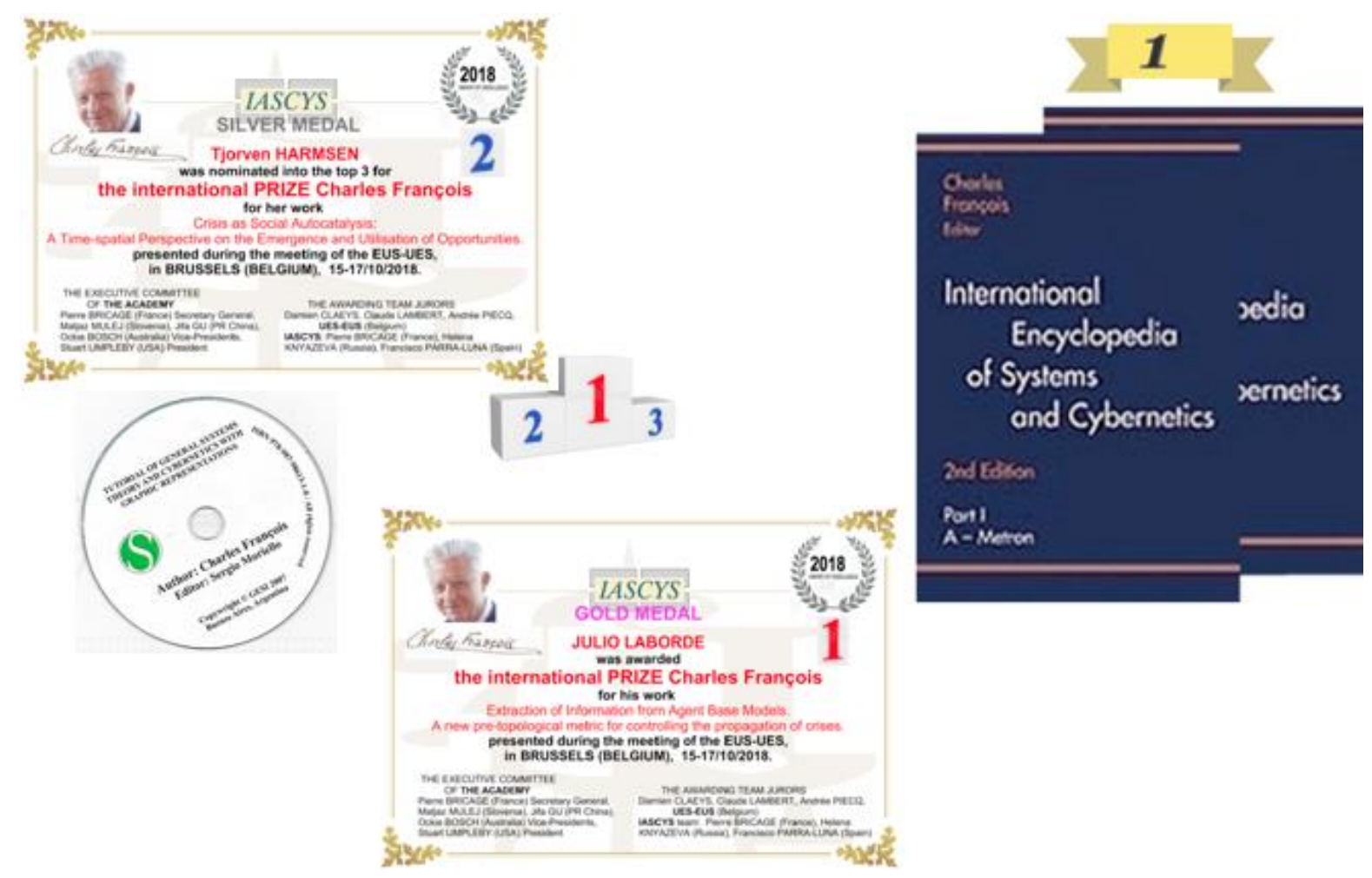

Figure 1. Charles François International Prize: awards and certificates.

Finally 3 second places ( 3 silver medals) were awarded, but only 1 Prize (gold medal, rank 1 ). All recipients got the CD of the Charles François tutorial in Systems Science, on a virus-free USB stick. Only the $1^{\text {st }}$ place can get the 2 volumes of the Encyclopedia. The certificates indicate the rank ( $1^{\text {st }}$ or top 3$)$ and the title of the awarded paper/talk.

La génération "montante" a été particulièrement remarquée, avec la remise du prix (médaille d'or) à Julio LABORDE et d'un accessit (médaille d'argent) à Mademoiselle Tjorven HARMSEN.

- Julio LABORDE ${ }^{9}$, qui a remporté cette première édition du prix, est un jeune chercheur Chilien, qui est ingénieur chez 'Insight-Signals' et aussi étudiant à l'Ecole Pratique des Hautes Etudes à Paris,

9 Julio LABORDE julio.laborde@insight-signals.com

Julio Laborde grew in Santiago de Chile, where he got a degree on Mathematics at the Universidad Catolica. In 2012 he moved to France, where he got a masters degree on Complex Systems, about natural cognition and artificial intelligence, at the EPHE. Then he decided to pass a PhD on Complex Systems in the same School. His research is mostly focused on graph 
France. Sa communication primée, présentée dans le cadre de la session 'Methods and tools for risk management of complex socio-technical systems' portait sur 'Extraction of Information from Agent Base Models. A new pre-topological metric for controlling the propagation of crises.' Elle est représentative à la fois d'une réflexion personnelle et d'un travail au sein d'une équipe associant des structures publiques et privées..

- Tjorven HARMSEN ${ }^{10}$, qui a obtenu un accessit (médaille d'argent), est une très jeune suédoise, étudiante en $\mathrm{Ph} \mathrm{D}$, en Allemagne, au Leibniz Institute for Research on Society and Space, près de Berlin, dont le travail portait sur 'Crisis as Social Autocatalysis. On the emergence and Utilization of Opportunities', dans la session 'Crises, Cybersystemic Management of Changes'.

A compétence et qualité égales, la préférence a été donnée à ces jeunes, afin de les encourager dans la voie très difficile de la transdisciplinarité.

\section{CONCLUSION}

Après un processus de pré-sélection anonyme sur le contenu du résumé et d'évaluation "sévère" en situation de congrès, le PRIX Charles François -médaille d'or- a été attribué à Julio LABORDE (Chili) et des accessits, 3 médailles d'argent ex-aequo, ont été attribués à Mick ASBY (Germany), Tjorven HARSMEN (Germany) et Daniela TERRILE (Italy). Le Prix récompense donc à la fois des chercheurs confirmés 'oubliés' et de jeunes chercheurs 'prometteurs', qui s'épanouissent ou/et s'épanouiront dans des domaines multi-culturels, multi-lingues, trans-disciplinaires, comme l'a fait, en précurseur, Charles FRANÇOIS.

\section{RÉFÉRENCES}

Bricage, P. (2014). "The International Academy for Systems and Cybernetic Sciences IASCYS". Rev. Intl. de Sistemas, 19: 01-04. https://www.uv.es/sesgejd/RIS/19/1.Bricage.IASCYS.pdf

Bricage, P. (2017). "Teaching \& Learning Systems Thinking: What, Why, When, Where, Who, What For, How?“ Rev. Intl de Sistemas, 21(1): 9-16. https://ojs.uv.es/index.php/ris/article/view/11598/10943 François, Ch. (2007). Tutorial of General Systems Theory and Cybernetics with Graphic Representations. 1 CD. Moriello, S. Editor, GESI, Buenos Aires, Argentina.

Francois, Ch. Editor (2004). The International Encyclopedia of Systems and Cybernetics, Creative Common International Team (ALAS, IASCYS, IFSR), K.G. Saur, München, Germany, 2nd edition, 2 vol., $741 \mathrm{p}$.

Collectif JMT (28 septembre 2018). Journée Mondiale de la Traduction, Pau, France, Europe.

Ronen, S. et al. (2014). "Links that speak: The global language network and its association with global fame”. PNAS 111(52): E5616-E5622. http://www.pnas.org/content/111/52/E5616

\footnotetext{
theory and pre-topology as tools for the study of Complex Systems. At the same time he is working about social modelling for the Insight-Signal Company.

10 Tjorven HARMSEN tjorven.harmsen@leibniz-irs.de

Tjorven Harmsen is a doctoral researcher at the Leibniz Institute for Research on Society and Space (IRS) in Erkner near Berlin. She works in an empirical project on crisis management and the role of expert advice in resilience activation processes. Studying sociology at the universities of Bremen and Jena, Germany, she developed particular interest for systems research. With a focus on difference-theoretical findings of Niklas Luhmann, she explored forms of the co-evolution of psychic and social systems. To deepen her understanding of system-environment relations and different modes of observation, she since then has participated in several activities in the Evolution, Complexity and Cognition (ECCO) research group, Brussels, Belgium.
} 